\title{
Identification of High-Risk Patients in the Pediatric Emergency Department with Symptoms of Headache
}

\author{
Funda Kurt ${ }^{1, \star}$, Halil İbrahim Yakut ${ }^{1}$
}

\author{
${ }^{1}$ Division of Pediatric Emergency \\ Medicine, Ankara City Hospital, Ankara, \\ Turkey \\ *Correspondence \\ drfundakurt@gmail.com \\ (Funda Kurt)
}

\begin{abstract}
Objective: It is vital to detect life-threatening conditions in patients presenting to the pediatric emergency department with symptoms of headache. In this study, we aimed to evaluate patients who presented to our pediatric emergency department with symptoms of headache, and to determine which findings deserved the most attention in the anamnesis and during physical examinations.

Methods: Patients who presented to the pediatric emergency department with symptoms of headache between July 2018 and June 2019 in Ankara, Turkey, were evaluated, retrospectively. Results: During the study period, 743 patients were included in the study. The median (IQR) age was 160.0 (range, 20.0192.0) months. The male and female distribution was $46.2 \%(\mathrm{n}=343)$ and $53.8 \%(n=400)$, respectively. The most common cause of headache was upper respiratory tract infections $(84.2 \%)$. Severe headache symptoms were present in $2.3 \%$ of the entire patient group. In $81.2 \%$ of the patients with severe headache, various pathologic neurologic findings such as diffuse headache, nocturnal awakenings, vomiting, visual disturbances, nystagmus, cranial nerve pathologies, weakness, altered mental status, speech difficulty, and meningismus were present. Seventy-nine (10.6\%) patients underwent neurologic imaging, and $15(19.0 \%)$ had pathologic findings. Conclusion: Our data support that the most common cause of headaches in the pediatric emergency department is upper respiratory tract infections. The co-existence of nocturnal awakenings and/or the presence of pathologic-neurologic findings are red flags for cases of severe headache.
\end{abstract}

\section{Keywords}

Children, Headache, Pediatric emergency medicine

\section{Introduction}

Headache is one of the most frequent causes of admission to pediatric emergency departments (PED) $[1,2]$. The prevalence of headaches among children varies between $5.9 \%$ and $37.7 \%$, and the incidence increases with age [2]. Thirty to ninety percent of adolescents may present with headache symptoms at any time during this period [3].

According to the International Classification of Headache Disorders (ICHD-III beta version), headaches are classified as primary and secondary [4]. The most common primary headache causes in childhood are migraine and tension-type headache. Secondary headaches are directly associated with another clinical condition (i.e. infections, vascular or nonvascular intracranial disorders, or psychiatric disorders), and they usually recover after definite diagnosis and proper treatment of the underlying cause. Secondary headaches in childhood mostly have a benign etiology, and serious organic causes are seldom encountered (4-20\%) [2, 5-9].

In the emergency department environment, rapid clinical evaluation of patients and early detection of life-threatening situations are vital $[2,10,11]$. In order to achieve this, detailed physical examinations and funduscopic evaluation findings are critical to exclude serious conditions [12]. In funduscopic evaluation, papillary edema is a red-flag 
TA B LE 1. Characteristics of the patients with a headache in the pediatric emergency department.

n (\%)

\begin{tabular}{lc}
\multicolumn{1}{c}{ Age (months)* } & n (\%) \\
\hline Preschool children & $160.0(120.0-192.0)$ \\
\hline School-age children & $49(6.6)$ \\
Adolescents & $311(41.9)$ \\
Sex & $383(51.5)$ \\
Male & $343(46.2)$ \\
Female & $400(53.8)$ \\
Type of admission to the hospital & \\
EMS & $16(2.2)$ \\
With their own means & $727(97.8)$ \\
\hline Headache duration time (hour) & $8.0(6.0-12.0)$ \\
\hline PED observation & $77(10.4)$ \\
\hline PED follow-up time (hour) & $12.0(8.0-24.0)$ \\
\hline Location & \\
\hline Localized pain & $602(81.0)$ \\
\hline Diffuse pain & $34(4.6)$ \\
\hline Region not mentioned & $107(14.4)$ \\
\hline
\end{tabular}

* median (IQR)

EMS: Emergency medical service, PED: Pediatric emergency department, IQR: Interquartile range.

finding [5]. In emergency situations, the patient should be evaluated using computed tomography (CT) scanning and/or magnetic resonance imaging (MRI).

In this study, we retrospectively analyzed the clinical and radiologic findings of patients who were admitted to our PED with symptoms of headache. Our purpose was to determine red-flag findings in cases of severe headache.

\section{Methods}

This retrospective study was conducted between July $1^{\text {st }}$, 2018, and June $30^{t h}, 2019$, in the PED of Ankara Child Health and Diseases Hematology Oncology Training and Research Hospital, University of Health Sciences, Ankara, Turkey. Our hospital is a tertiary care hospital that serves approximately 140,000 patients annually. A computerized data search of patients aged between 3 and 18 years who received the code 'R51-Headache' [the International Classification of Diseases Version 10 (ICD-10 coding system)] resulted in a total of 812 patients. Forty-one patients with a wrong diagnostic code, 25 with inadequate information, and three patients with ventriculoperitoneal shunt were excluded from the study.

The study was planned according to the principles of the Declaration of Helsinki and the study protocol was approved by the Hospital Ethics Committee (No. E1/107/2019)

Age, sex, family history of migraine, duration of symp- toms, associated symptoms, location of pain, sleep interruptions due to headache, presence of abnormal neurologic findings, the length of stay in the PED observation unit, neuroimaging study findings if available, and the final outcome were recorded and analyzed retrospectively from the hospital records.

The type of hospital transfer was also studied. In Turkey, a standard phone number (112) is used to request on-site emergency healthcare. A fully equipped ambulance with one or two paramedics (sometimes including a physician) goes to the request address, and in case of necessity, the patient's transfer to the emergency department is provided. In the present study, some of the patients in our sample used the ambulance service, the others were taken to hospital by their relatives.

The patients were grouped according to their age at the time of admission as preschool (3-5 years), school-age (612 years), and adolescent (13-18 years) [2].

Headache was defined as primary or secondary, according to the ICHD-III beta criteria [4]. Patients with one of the following: organic and structural involvement of the central nervous system, requirement of any emergency diagnostic test, and/or a necessity for immediate intervention in the PED were considered as 'severe headache'.

\subsection{Statistical analysis}

Statistical analysis was performed by using the Statistical Package for the Social Sciences (SPSS) v.20 (SPSS Inc., Chicago, IL, USA) program. The normality of data distribution was evaluated using the KolmogorovSmirnov normality test. Normally distributed variables are expressed as mean \pm standard deviation (SD), and non-normally distributed variables are expressed as median [interquartile range (IQR)]. The Mann-Whitney $\mathrm{U}$ test was used to compare non-normally distributed variables between two groups. The Pearson Chi-square test was used to compare categorical variables. A p-value of $<0.05$ was considered statistically significant. A Kappa coefficient was used to evaluate the concordance between the diagnoses at the time of first admission and at emergency readmissions.

\section{Results}

During the study period, a total of 125,334 patients presented to the PED. Seven hundred seventy-one $(0.6 \%)$ patients were admitted with symptoms of headache. Of these, 743 patients were included in the study. The median (IQR) age was 160.0 (120.0-192.0) months, and 343 (46.2\%) patients were male. In the adolescent age group, the male and female distribution was $38.9 \%(n=149)$ and $61.1 \%(n$ $=234)$, respectively $(\mathrm{p}<0.001)$.

A total of 77 (10.4\%) patients were followed up in the PED observation unit. The mean follow-up time was median (IQR) 12.0 (8.0-24.0) hours, and the median (IQR) headache duration was $8.0(6.0-12.0)$ hours (Table 1). The most frequent cause of headache in the entire group was 
TA B L E 2. Causes of headaches in the pediatric emergency department.

\begin{tabular}{|c|c|c|}
\hline \multicolumn{2}{|c|}{ Number of patients } & Prevalence among total headaches (\%) \\
\hline \multicolumn{3}{|l|}{ Causes of primary headaches } \\
\hline Migraine & 22 & 3 \\
\hline Tension-type headache & + & 0.5 \\
\hline \multicolumn{3}{|l|}{ Causes of secondary headaches } \\
\hline Trauma & 1 & 1.5 \\
\hline Toxic exposure* & 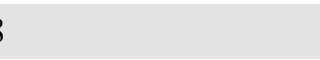 & 1.1 \\
\hline Infection & 6 & 84.2 \\
\hline Neurologic disorders & 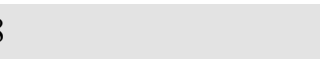 & 1.1 \\
\hline Hypertension & 4 & 5.9 \\
\hline Others ${ }^{* *}$ & 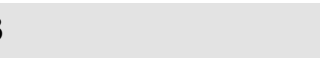 & 0.4 \\
\hline Severe conditions & 7 & 2.3 \\
\hline Total number of headaches & 13 & \\
\hline \multicolumn{3}{|c|}{$\begin{array}{l}\text { * Substance abuse, carbon monoxide poisoning. } \\
\text { ** Drug adverse effects (intravenous immunoglobulin, cycloplegic eye drops) and after prolonged starvation. }\end{array}$} \\
\hline Diagnosis & Number of patients & Prevalence among total headaches $(\%)$ \\
\hline Brain tumors & 5 & 0.7 \\
\hline Intracranial infections & 2 & 0.3 \\
\hline meningitis & 2 & \\
\hline Intracranial vascular disorders & 2 & 0.3 \\
\hline moyamoya disease & 1 & \\
\hline cerebral venous sinus thrombosis & 1 & \\
\hline Others & 8 & 1 \\
\hline idiopathic intracranial hypertension & 3 & \\
\hline pineal cyst & 2 & \\
\hline neuromyelitis optica & 1 & \\
\hline facial and vestibular nerve neuritis & 1 & \\
\hline Chiari malformation type 1 & 1 & \\
\hline Total number of severe headaches & 17 & \\
\hline
\end{tabular}

hypertension (39.0\%). Other less common causes were upper respiratory tract infection $(19.5 \%)$, neurologic disorders (e.g. seizures) (11.7\%), serious illness (e.g. brain tumors, intracranial vascular disorders) $(13.0 \%)$, intoxication $(9.1 \%)$ (e.g. substance abuse, carbon monoxide poisoning), head trauma (1.5\%), and migraine $(2.6 \%)$. Two $(2.6 \%)$ other patients were in the study group, one of whom had a headache after using cycloplegic eye drops, and the other patient had combined immune deficiency and underwent intravenous immunoglobulin (IVIG) treatment 8 hours prior to PED admission.

During the study period, $715(96.2 \%)$ patients had only one PED admission, $26(3.5 \%)$ patients had a second consecutive PED application with the same symptoms. Two $(0.3 \%)$ patients visited three times with a median 62.9 (range, 0-230) days between consecutive PED presenta- tions. In 10 (35.7\%) patients, re-admission to the PED was recorded within five days. The diagnoses in this group were similar to the previous diagnoses in the majority of patients $(\kappa=0.705, \mathrm{p}<0.001)$.

Of the 743 patients, $26(3.5 \%)$ were diagnosed as having primary headache (migraine and tension-type), and 717 $(96.5 \%)$ had a secondary headache. The median (IQR) age of patients with primary headache was 190.5 (170.5$210.0)$ months, and eight $(30.8 \%)$ were male. The median (IQR) age of patients with secondary headache was 157.0 (117.5-191.0) months, and 335 (46.7\%) were male. Primary headache was mostly seen in older children ( $p$ $<0.001)$. There was a family history of migraine in 64 $(8.6 \%)$ patients. Similarly, nine $(34.6 \%)$ patients who were evaluated as having primary headaches had a history of migraine in the family. 
TA B L E 4. Comparison of symptoms and physical examination findings in severe and non-severe headaches.

\begin{tabular}{|c|c|c|c|}
\hline & Severe headache $(\mathrm{n}, \%)$ & Non-severe headache $(\mathrm{n}, \%)$ & p-value \\
\hline Location & & & $<0.001^{*}$ \\
\hline Localized pain & $13(76.5)$ & $604(83.2)$ & \\
\hline Diffuse pain & $4(23.5)$ & $31(4.3)$ & \\
\hline Region not mentioned & $0(0.0)$ & $91(12.5)$ & \\
\hline No headache history & $12(75.0)$ & $544(75.8)$ & 0.999 \\
\hline \multicolumn{4}{|c|}{ Symptom or physical examination findings } \\
\hline Vomiting & $8(50.0)$ & $104(14.3)$ & $0.001^{*}$ \\
\hline Nocturnal awakenings & $3(18.8)$ & $11(1.5)$ & $0.003^{*}$ \\
\hline Pain relief with analgesic & $15(93.8)$ & $718(98.8)$ & 0.197 \\
\hline Visual disturbances & $5(31.2)$ & $6(0.8)$ & $<0.001^{*}$ \\
\hline Pupillary abnormalities & $1(6.2)$ & $0(0.0)$ & $0.022^{*}$ \\
\hline Strabismus & $1(6.2)$ & $0(0.0)$ & $0.022^{*}$ \\
\hline Nystagmus & $2(12.5)$ & $0(0.0)$ & $0.001^{*}$ \\
\hline Dizziness & $0(0.0)$ & $41(5.6)$ & 0.999 \\
\hline Ataxia & $1(6.2)$ & $1(0.1)$ & $0.043^{*}$ \\
\hline Paresthesia & $3(18.8)$ & $18(2.5)$ & $0.009^{*}$ \\
\hline Cranial nerve palsy & $3(18.8)$ & $1(0.1)$ & $<0.001^{*}$ \\
\hline Weakness & $2(12.5)$ & $1(0.1)$ & $0.001^{*}$ \\
\hline Altered mental status & $5(31.2)$ & $9(1.2)$ & $<0.001^{*}$ \\
\hline Speech difficulty & $3(18.8)$ & $6(0.8)$ & $0.001^{*}$ \\
\hline Meningismus & $2(12.5)$ & $0(0.0)$ & $0.001^{*}$ \\
\hline
\end{tabular}

Column percentages are provided in the table.

The most common cause of secondary headache was viral or bacterial infections (especially upper respiratory tract), comprising $87.3 \%$ of secondary headaches. Other causes in this group were hypertension $(6.1 \%)$, toxic exposure [(carbon monoxide poisoning $(0.8 \%)$, and substance abuse $(0.3 \%)]$, post-seizure headache, and other severe conditions (Table 2). Severe headache was diagnosed in 17 patients. A brain tumor was detected in $5(0.7 \%)$ patients, and two patients had intracranial infections $(0.3 \%)$, two patients $(0.3 \%)$ had intracranial vascular disorders, and three $(0.4 \%)$ had idiopathic intracranial hypertension (Table 3$)$.

We also analyzed the location of the pain, previous headache history, accompanying symptoms, and physical examination findings. Diffuse headache $(p<0.001)$, vomiting $(p=0.001)$, nocturnal awakenings with headache $(\mathrm{p}=0.003)$, visual disturbances $(\mathrm{p}<0.001)$, pupillary abnormalities $(p=0.022)$, strabismus $(p=0.022)$, nystagmus $(\mathrm{p}=0.001)$, ataxia $(\mathrm{p}=0.043)$, paresthesia $(\mathrm{p}$ $=0.009)$, cranial nerve palsy $(\mathrm{p}<0.001)$, weakness $(\mathrm{p}=$ 0.001 ), altered mental status $\mathrm{p}<0.001$ ), speech difficulty $\mathrm{p}=0.001$, and meningismus findings $(\mathrm{p}=0.001)$ were more common in severe headaches (Table 4 ).

One hundred twenty-five $(16.8 \%)$ patients were referred to other clinics, including pediatric neurology $(n=42)$, neurosurgery $(n=20)$, pediatric nephrology $(n=37)$, ophthalmology $(n=16)$, and ten other departments. Neuroimaging studies were performed in 79 patients, which comprises $10.6 \%$ of patients. Of those, $36(4.8 \%)$ patients had cranial CT, 43 (5.8\%) had MRI, and five patients had both brain CT and MRI. The rate of abnormal findings was $25.3 \%(n=20)$; $15(19.0 \%)$ patients had pathologic findings such as brain tumor, infarction in the cerebral hemispheres, abnormal moyamoya vessels, venous infarction, venous thrombosis, pineal cyst, and herniation of cerebellar tonsils. The other five $(6.3 \%)$ patients had incidental benign abnormalities not related to the reported symptoms (arachnoid cysts without hydrocephalus).

In patients with and without pathologic clinical examination findings, positive neuroimaging results were $52.4 \%$ and $0.4 \%$, respectively $(\mathrm{p}<0.001)$ (Table 5 ). In patients with and without severe headache, the rate of pathology in the neurologic examination was $87.0 \%$ and $1.1 \%$, respectively $(p<0.001)$. Neurologic examinations were considered as normal in three $(13.0 \%)$ patients with severe headache, but one of these patients had projectile vomiting, and two had nocturnal awakenings with headache. 
TA B L E 5. Comparison of neurologic examination and neuroimaging results.

\begin{tabular}{lccc}
\multicolumn{4}{c}{ Neurologic Examination } \\
& Normal n, \% & Pathologic n, \% & Total n, \% \\
\hline Neuroimaging & $53(7.3)$ & $7(33.3)$ & $59(7.9)$ \\
Normal & $3(0.4)$ & $11(52.4)$ & $15(2.0)$ \\
Pathologic & $5(0.7)$ & $0(0.0)$ & $5(0.7)$ \\
\hline Incidental benign abnormality & $661(91.6)$ & $3(14.3)$ & $664(89.4)$ \\
Not performed & $722(100.0)$ & $21(100.0)$ & $743(100.0)$ \\
\hline Total & & \\
\hline
\end{tabular}

*Column percentages are provided in the table.

\section{Discussion}

Headache is a common symptom in children. In our study, we found that secondary headache was more common in children, and the most common secondary cause was upper respiratory infection. Severe headache was rare, comprising only $2.3 \%$ of the cases. Diffuse headache, vomiting, visual disturbances, pupillary abnormalities, nocturnal awakening with headache, strabismus, nystagmus, ataxia, paresthesia, cranial nerve palsy, weakness, altered mental status, speech difficulty, and meningismus findings were more prevalent in cases of severe headache.

The proportion of patients admitted to the PED with symptoms of headache $(0.6 \%)$ in our series was similar to prior studies, representing a range of $0.6-1.3 \%$ of PED visits $[1,2,5,6,12,13]$.

In some studies, headache was found to be more common in the male sex (50.3-62.3\%) [1, 2, 5, 6, 8]. Glatstein et al. [12] evaluated 295 cases of headache retrospectively and reported that the rate of male sex was $44 \%$. Our series also represents a lower rate $(46.2 \%)$. This result may result may be due to the higher percentage of patients in the adolescent age group in our study, in which there was a female preponderance.

The average age of children with headache was found as 8.8-9.6 years in previous studies [1, 2, 5, 6, 8]. Glatstein et al. [12] reported that the mean age and median age were 10.2 and 10.4 years, respectively. In our study, the median age was 13.3 years. The relatively higher median age in our series may represent the exclusion of patients aged under 3 years, while in other studies, children aged under 2 years were also included.

In the PED setting, early detection of dangerous and reversible causes of headache have critical importance. The first step is to distinguish primary (e.g. migraine, cluster headaches) and secondary (i.e., due to an underlying cause such as infection, mass lesion) cases [12]. The frequency of primary headache was reported as $23.8-55 \%$ in adults $[14,15]$ and $10-24.5 \%[2,5]$ in the pediatric age group. Various studies reported that secondary headaches were more common in children with upper respiratory tract infections [2, 5, 6, 8, 16]. Parallel to other studies in the literature, we found that $96.5 \%$ of our patients had secondary type headache. Respiratory infections comprised
$87.3 \%$ of these cases. It was reported that headache was more common in school-aged children, where respiratory tract infections commonly occur [2, 5, 6, 8]. In our study, we found that $41.9 \%$ of the patients were school-age.

Primary headache was reported to be more common in older children and in girls $[1,17]$. Caperell et al. [17] found that $64.7 \%$ of patients with primary headache were female. Similarly, our results support that the primary headache cases were predominant in the older age (median age 190.5 months) and female sex (69.2\%) group.

The rate of return visits to the emergency department (RTED) caused by headaches was $4.4 \%$ and $9.0 \%[1,6]$. Scagni et al. [6] reported that $56.5 \%$ of the patients were due to primary treatment failure, and $46.5 \%$ had a new headache episode as the reason for RTED within a week. Rossi et al. [1] reported that among patients who returned within ten days, $78.5 \%$ had persistent headaches, $11.2 \%$ revealed new symptoms (e.g. vomiting, fever), and $8.1 \%$ had a new headache episode. We determined that $3.8 \%$ of our cases were RTED due to headache. Of these readmissions, $35.7 \%$ were within five days, and the final diagnosis was similar to the primary decision.

Most secondary headaches are benign and self-limiting. It has been reported that the rate of severe life-threatening conditions in headaches in this group was 3.3-15.3\% [1, 2, $5,16]$. Similar to these studies, the rate of severe headache was $2.3 \%$ in our series.

Regarding severe headache, Sobri et al. [18] stated that there were five red-flag symptoms such as papilledema, drowsiness, paralysis of extremities, asymmetrical pupillary response, and progressive loss of vision or neurologic instability. Cain et al. [13] reported that visual and sensory changes, fatigue, speech difficulty, altered mental status, abnormal strength, abnormal reflexes, coordination, and gait, and cranial nerve palsy were serious findings that necessitated immediate neuroimaging studies. Conicella et al. [2] showed that the presence of focal neurologic deficits, papilledema, ataxia, and consciousness changes increased the risk of life-threatening complications in these cases. Rossi et al. [1] found at least one clinical warning indicator in $79.2 \%$ of children with severe headache and concluded that visual impairment, cranial nerve palsies, pupillary abnormalities, nystagmus, ataxia, hyposthenia, 
strabismus, drowsiness, and meningismus symptoms were mostly associated with severe headache. In our study, diffuse headache, vomiting, night-awakening headache, visual impairment, pupillary abnormalities, strabismus, nystagmus, ataxia, paresthesia, cranial nerve palsy, weakness, altered mental status, speech difficulty, meningismus findings were found more commonly in severe headaches compared with the rest of the group.

Headache is the second most common symptom in patients undergoing cranial CT imaging, and the most common symptom in patients undergoing MRI in emergency care centers [19, 20]. Despite this fact, there are no widely accepted clinical pathways about when to perform imaging in patients admitted to the emergency department with acute-onset headaches. Several studies and the current American Academy of Neurology (AAN) guidelines state that neuroimaging is not recommended if the neurologic examination is normal in non-traumatic pediatric headaches $[3,21,22]$. Intracranial findings were detected in less than $5 \%$ of the imaging evaluations of patients with normal neurologic examinations $[2,5,6,9]$. Similar to the literature, in our study, neuroimaging was performed in $8 \%$ of the patients with a normal neurologic examinations, and only $0.6 \%$ of the imaging revealed pathologic findings.

Neuroimaging (cranial CT or MRI) rates in children with headaches range between $6.3-44 \%$ in different studies $[5,6$, $13,23,24]$. Broder et al. [25] evaluated the use of CT in a PED through a 6-year period and reported a $23 \%$ increase in cranial CT requests, despite a very small increase in patient admissions (2\%). Cain et al. [13] reported that the neuroimaging rate was $18 \%$, and the clinically significant finding rate was 3.8\%. In their series, Kan et al. [5] found that the rate of imaging and the presence of pathologic findings was $53 \%$ and $10 \%$, respectively. In secondary neurologic headache cases, these numbers were $33 \%$ and $0 \%$, respectively. Rossi et al. [1] reported that their neuroimaging rates were $8.8 \%$ and $12.5 \%$ in patients with abnormal findings $(7.1 \%$ had pathologic findings, $5.4 \%$ had non-relevant benign abnormalities). In our study, the neuroimaging rate $(10.6 \%)$ was compatible with the literature. The total abnormality detection rate was $25.3 \%$, of which $19.0 \%$ had relevant pathologic findings, and $6.3 \%$ had incidental benign abnormalities.

\section{Conclusion}

Despite the relatively low severe headache rate in children, proper identification of high-risk patients is vital. Detailed history taking and complete neurologic evaluation are of paramount importance in patients. Our study results suggest that the presence of symptoms such as diffuse headache, nocturnal awakening headache, vomiting, visual disturbances, pupillary abnormalities, strabismus, nystagmus, ataxia, paresthesia, presence of cranial nerve dysfunctions, weakness, altered mental status, speech difficulty, meningismus are red flags in cases of severe headache. More comprehensive studies involving a more substantial number of patients are needed to determine findings that may indicate high-risk and poor outcome cases of pediatric headache.

\section{Limitations}

Our study has some limitations, including its retrospective study design and the lack of complete follow-up monitoring. The reflection of a single-center experiment is another flaw. The analysis was limited to the information reported on the clinical records, and some incorrect or mistyped data might interfere with the findings. Additionally, there were only a few cases of severe headache, insufficient to draw statistically significant results for the proper selection of the risk factors.

\section{ACKNOWLEDGMENTS}

I would like to express my gratitude to all those who helped me during the writing of this manuscript.

\section{CONFLICT OF INTEREST}

Authors declare no financial or commercial conflict of interest.

\section{ETHICAL STATEMENT}

This article does not contain any studies on human or animal subjects performed by any of the authors.

\section{REFERENCES}

[1] Rossi R, Versace A, Lauria B, et al. Headache in the pediatric emergency department: A 5-year retrospective study. Cephalalgia. 2018;38:1765-72.

[2] Conicella E, Raucci U, Vanacore N, et al. The child with headache in a pediatric emergency department. Headache. 2008;48:1005-11.

[3] Sheridan DC, Meckler GD, Spiro DM, et al. Diagnostic testing and treatment of pediatric headache in the emergency department. The Journal of pediatrics. 2013;163:1634-7.

[4] Headache Classification Committee of the International Headache Society (IHS) The International Classification of Headache Disorders, 3rd edition. Cephalalgia. 2018;38:1-211.

[5] Kan L, Nagelberg J, Maytal J. Headaches in a pediatric emergency department: etiology, imaging, and treatment. Headache. 2000;40:25-9.

[6] Scagni P, Pagliero R. Headache in an Italian pediatric emergency department. J Headache Pain. 2008;9:83-7.

[7] Gandhi R, Lewis EC, Evans JW, et al. Investigating the necessity of computed tomographic scans in children with headaches: a retrospective review. Cjem. 2015;17:148-53.

[8] Hsiao HJ, Huang JL, Hsia SH, et al. Headache in the pediatric emergency service: a medical center experience. Pediatrics and neonatology. 2014;55:208-12.

[9] Lateef TM, Grewal M, McClintock W, et al. Headache in young children in the emergency department: use of computed tomography. Pediatrics. 2009;124:e12-7.

[10] Ward TN, Levin M, Phillips JM. Evaluation and management of headache in the emergency department. The Medical clinics of North America. 2001;85:971-85.

[11] Lewis DW, Scott D, Rendin V. Treatment of paediatric headache. Expert opinion on pharmacotherapy. 2002;3:1433-42.

[12] Glatstein M, Voliovitch Y, Orbach R, et al. Outpatient Management 
of Headache after Pediatric Emergency Department Visit: Are we Missing Anything? Headache. 2019;59:1530-6.

[13] Cain MR, Arkilo D, Linabery AM, et al. Emergency Department Use of Neuroimaging in Children and Adolescents Presenting with Headache. The Journal of pediatrics. 2018;201:196-201.

[14] Yu S, Liu R, Zhao G, et al. The prevalence and burden of primary headaches in China: a population-based door-to-door survey. Headache. 2012;52:582-91.

[15] Stovner L, Hagen K, Jensen R, et al. The global burden of headache: a documentation of headache prevalence and disability worldwide. Cephalalgia. 2007;27:193-210.

[16] Lewis DW, Qureshi F. Acute headache in children and adolescents presenting to the emergency department. Headache. 2000;40:200-3.

[17] Caperell K, Pitetti R. Seasonal variation of presentation for headache in a pediatric emergency department. Pediatric emergency care. 2014;30:174-6.

[18] M S, Lamont AC, Alias NA, et al. Red flags in patients presenting with headache: clinical indications for neuroimaging. The British journal of radiology. 2003;76:532-5.

[19] Larson DB, Johnson LW, Schnell BM, et al. Rising use of CT in child visits to the emergency department in the United States, 1995-2008. Radiology. 2011;259:793-801.

[20] Scheinfeld MH, Moon JY, Fagan MJ, et al. MRI usage in a pediatric emergency department: an analysis of usage and usage trends over 5 years. Pediatric radiology. 2017;47:327-32.
[21] Lewis DW, Dorbad D. The utility of neuroimaging in the evaluation of children with migraine or chronic daily headache who have normal neurological examinations. Headache. 2000;40:629-32.

[22] Lewis DW, Ashwal S, Dahl G, et al. Practice parameter: evaluation of children and adolescents with recurrent headaches: report of the Quality Standards Subcommittee of the American Academy of Neurology and the Practice Committee of the Child Neurology Society. Neurology. 2002;59:490-8.

[23] Eapen A, Agarwal R, Thomas R, et al. Management of pediatric migraine in a tertiary care versus community based emergency department: an observational pilot study. Pediatric neurology. 2014;50:164-70.

[24] DeVries A, Young PC, Wall E, et al. CT scan utilization patterns in pediatric patients with recurrent headache. Pediatrics. 2013;132:e1-8.

[25] Broder J, Fordham LA, Warshauer DM. Increasing utilization of computed tomography in the pediatric emergency department, 20002006. Emergency radiology. 2007;14:227-32.

How to cite this article: Funda Kurt, Halil İbrahim Yaku. Identification of High-Risk Patients in the Pediatric Emergency Department with Symptoms of Headache. Signa Vitae. 2020;16(1):115-121. doi:10.22514/sv.2020.16.0015. 